\title{
Hunting for Light Dark Matter with the NOvA Detector
}

\author{
Peter Filip, for the NOvA collaboration \\ Institute of Physics, Czech Academy of Sciences, Na Slovance 1999/2, Prague, 182 21, Czech Republic
}

\begin{abstract}
Direct detection of dark matter is the ultimate goal of experimental physics. NOvA Near detector may be capable to put an upper limit on the lightweight dark matter (LDM) particle production, in the 120-GeV proton-nucleus collisions, engaged in producing the high-intensity NuMI neutrino beam. We summarize the approach used in the search for the elastic, dark matter - electron scattering in the Near Detector, and briefly mention two other efforts to observe the elusive dark matter interactions, with the NOvA detectors.
\end{abstract}

Keywords: dark matter, elastic scattering, NOvA detector

DOI: 10.31526/ACP.NDM-2020.30

\section{INTRODUCTION}

Several indirect signatures suggest [1] our Universe contains a large amount of gravitationally interacting matter of unknown origin. Assuming dark matter constituents can interract via electroweak, or other [2] effective dark forces, scientists are looking for a direct experimental confirmation of the interaction of DM particles, with the stable leptons and nucleons, in detectors.

Theoretically, the interaction of DM with the Standard Model particles can be assumed to proceed via kinetic mixing [2] of DM mediator (dark photon) with the regular U(1) photon of QED. Since the promissing indications [3] for the seasonal variation of the Milky way DM halo interaction with the $\mathrm{NaI}(\mathrm{Tl})$ cryogenic detectors were not confirmed by other experiments, a possibility for the production of DM particles in the high-intensity neutrino beam facilities (Fermilab), and the search for the anomalous scattering events in detectors located near the intense neutrino beam, obtain a new boost of the interest. In the next section we briefly describe the approach used in the search for the anticipated elastic DM particle interactions with electrons, in the NOvA Near Detector.

\section{THE PRODUCTION AND SCATTERING OF LDM PARTICLES}

While searching for LDM particles using the neutrino detector, located in the vicinity of the high-intensity neutrino beam, one assumes the neutrino beam to be "contaminated" by an admixture of DM particles, originating in the target (see Fig. 1). Thus, one considers a flux of DM particles of magnitude $\varphi_{D M}$ which depends on the (unknown) parameters of DM production mechanism considered, and on the total intensity of the proton beam used to generate the neutrino flux $\varphi_{v}$ passing through the detector.

The flux $\varphi_{D M}$ of DM particles can interact with the protons or nuclei, or the electrons contained in the active volume of detector, with the probability depending on the (a priori unknown) DM elastic scattering cross-section. For the specific chosen (kinetic mixing) DM interaction model [4], the scattering cross section $\sigma(\chi, e)$ scales with the dark sector $\left(\alpha_{D} \varepsilon^{2} / m_{D P}^{2}\right)$ interaction parameters, and it may even exceed the neutrino-electron scattering cross-section by orders of magnitude [4].

The NOvA collaboration concentrates on the electron-DM interactions, also because the neutrino-electron interactions (which are the background for DM-electron elastic scattering signal) are believed to be well understood within the Standard Model. A significant deviation, from the expected neutrino - electron interactions, observed with the known neutrino beam flux $\varphi_{v}$ passing through the detector, may be considered as a signature of the anticipated dark matter contamination of the neutrino beam, provided all systematic effects are under control, and the neutrino sector of the Standard Model is completely understood.

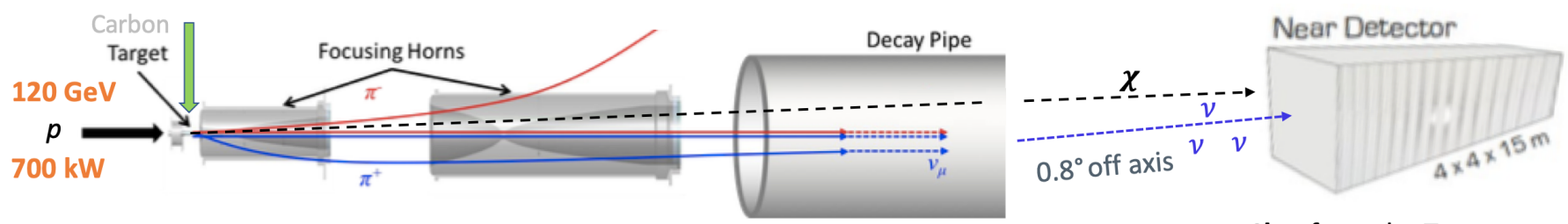

$675 \mathrm{~m}$ long

$1 \mathbf{k m}$ from the Target

FIGURE 1: Geometry of the NuMI neutrino beam generation, and of the anticipated DM production, with the magnetic focusing horns, the decay pipe, and the NOvA near detector shown. Dark matter is presumably produced in the target (see the green arrow). 


\subsection{Scattering of LDM Particles}

Elastic scattering of LDM particles on electrons is expected to generate a different angular distribution of the scattered electrons, if compared to the neutrino-electron scattering. In general, the reasons are quite simple: the spin of the generated neutrinos is exclusively longitudinally polarized, and neutrinos are known to have very small rest masses. On the contrary, the LDM particles can be scalar (zero spin) or unpolarized fermionic objects, substantially more massive than the neutrinos, coupled to the dark photon (DP) of sub-GeV mass: $m_{D P}$. The angular distribution of electrons in $v_{\mu}-e$ scattering events, in the NOvA detector, is predicted to be forward peaked, with the majority of events fulfilling the condition

$$
\theta_{e}^{2} E_{e}[\mathrm{GeV}]<0.005,
$$

where $\theta_{e}$ is the electron scattering angle in radians. The observed electron-type showers, with the reconstructed electron energy $E_{e}$, not fulfilling Eq.(1), belong to the sideband angular region of the neutrino-electron scattering. This region is assumed be the "signal" region for DM-electron scattering events, since the excess of the electron showers due to LDM interactions is anticipated there. As shown in Fig. 2 (left panel), obtained using the MC simulation [5], the sideband angular region contains the electron showers of neutrino-induced origin, which constitute the background in the search for LDM scattering signal. Therefore, a precise MC simulation, and detailed knowledge of the neutrino beam (intensity, energy spectrum, and flavour composition) are crucial.

\subsection{Production of LDM Particles}

In Fermilab, the high-intensity neutrino beam is generated via leptonic $\left(\pi^{ \pm}, K^{ \pm} \rightarrow v+l^{ \pm}\right)$decays of the charged mesons, created in the proton - nucleus interactions, within the thick target. The proton beam of energy $120 \mathrm{GeV}$, extracted from the FNAL Main injector, generates myriads of charged and neutral mesons in the carbon target, with the charged mesons being focused by the pulsed "horn" magnetic fields, in order to create a concentrated flux of neutrinos in a desired direction (see Fig. 1).

The production of LDM particles is anticipated to occur mainly within the target, whenever real or virtual photons are created, via the bremsstrahlung, neutral $\pi^{0}, \eta \rightarrow \gamma \gamma$ decays, or via Drell-Yan type $\left(q \bar{q} \rightarrow \gamma^{*} \otimes V_{D M} \rightarrow \chi \chi^{\dagger}\right)$ process. Kinetic mixing [2] of the QED photon $\gamma$ with the DM vector mediator $\left(A^{\prime}\right.$ or $\left.V_{D M}\right)$, is supposed to allow for the LDM production [4], with the probability depending on the LDM particle mass, mixing parameter $\varepsilon^{2}$, and dark photon mass, $m_{D P}$.

Since the NuMI neutrinos [6] are produced at a significant distance from the target (see Fig. 1), at the region where the majority of the focused charged mesons decay (in the $675 \mathrm{~m}$ long decay pipe), and because the NOvA Near Detector is shifted off the neutrino beam axis (by 14mrad), there is a small relative angle difference, between the effective source of the neutrinos and the anticipated source of LDM particles - the NuMI target (when viewed from the NOvA detector). This angle difference, however, is too small for the angular separation of the (beam) neutrino interactions, from the elastic scattering of LDM particles with electrons, in the detector. "Off-target" LDM production may also occur in the NuMI hadron absorber [6], or in the decay pipe, from the charged kaon decays $K^{ \pm} \rightarrow \pi^{ \pm}+\pi^{0} \rightarrow \gamma V_{D M}$ (via kinetic mixing), or e.g. from the very rare $K_{L}^{0} \rightarrow v \bar{v}+X_{D M}$ decays [7].

The energy distribution of the produced LDM particles can be simulated, for a given DM production model, and depending on the DM parameters used, one can try to locate [8] the region of the electron shower energies (e.g. high-recoil $E_{e}>5 \mathrm{GeV}$ region), where the excess, originating from the LDM - electron interactions, may be observable (see Fig. 2, right panel). Similar considerations in this direction can be found in [9]. A possibility to separate the LDM-electron scattering events from the neutrino interactions, using the precise timing and the high-frequency RF bunch structure considered [10] for the primary proton beam, is not applicable for the NOvA detector, since such approach requires a sub-nanosecond detector time resolution.

\section{NOvA Simulation}

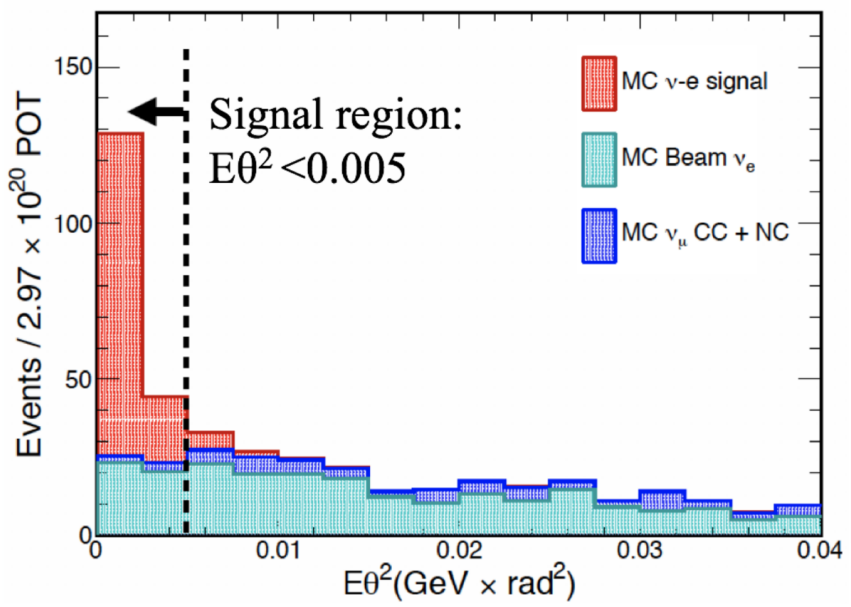

NOvA Simulation

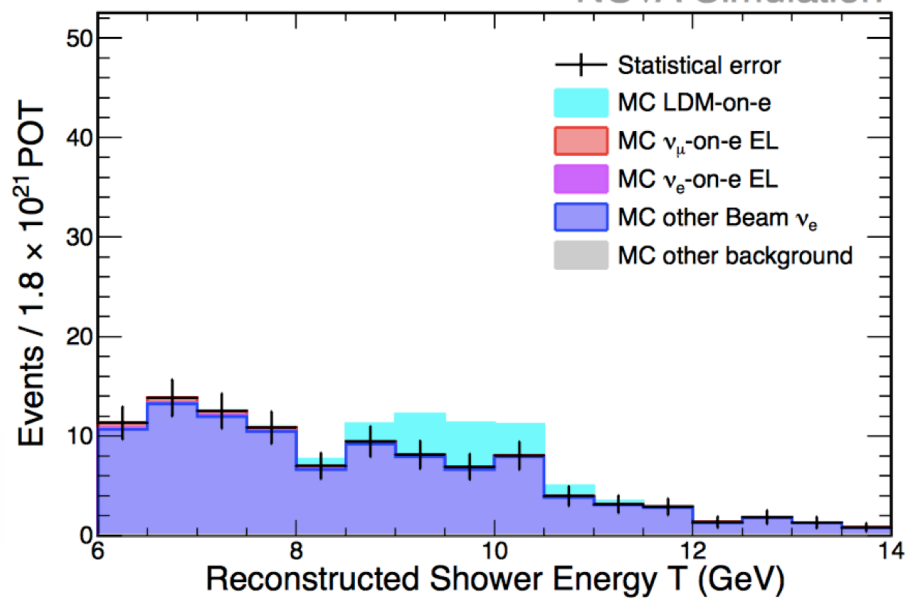

FIGURE 2: Left panel: Distribution of the showers, simulated for the NuMI neutrino beam interactions, in the NOvA Near detector [5]. Right panel: Example of the excess, due to DM-electron scattering events [8], on top of the neutrino-induced shower spectrum. 


\section{UPPER LIMITS ON LDM PRODUCTION}

If the neutrino beam flavour composition, intensity, and the energy spectrum (at the detector location) are not known with a sufficient precission, the signature of LDM-electron scattering, in the electron-like shower (angular and energy) spectrum, cannot be separated from the neutrino-initiated processes. The publication [4] assumes $10 \%$ uncertainity in the neutrino beam intensity, and estimates the upper limits on the LDM production for the NOvA detector, in $\left\{m_{\chi} ; Y=\varepsilon^{2} \alpha_{D}\left(m_{\chi} / m_{D P}\right)^{4}\right\}$ parameter space (see Figs. 1, 2, in ref. [4]). If a statisticaly significant excess of the electron-like showers, above the MC simulation, is not observed in any particular angular or energy region, the upper limit on the parameters of a given LDM model can be obtained, by scaling the LDM parameters up to the range, where the elastic DM-electron interactions (estimated by the MC simulation) would match two standard deviations $(2 \sigma)$ of the observed electron shower multiplicity, in a given energy and angular region [4].

Our description, of determining the upper limit on the LDM production in the NOvA experiment, is very simplified here. We have skipped many substantial details of the sophisticated effort necessary for separating the single electron showers from other event topologies. Moreover, just a reliable determination of the electron shower direction, and the estimate of the most probable electron energy, from the measured shower energy and topology, are non-trivial tasks (not described here).

NOvA collaboration intends to accumulate the neutrino-induced data until 2025, with the increased (currently $700 \mathrm{~kW}$ ) NuMI beam intensity, in the both: neutrino and anti-neutrino production modes. The full statistics obtained should allow one to put the limits [4] on the parameters of a given Light Dark Matter (production and interaction) models chosen.

\section{OTHER DM SEARCHES WITH NOVA DETECTORS}

NOvA experiment is taking data with two functionally identical detectors, separated from each other by $810 \mathrm{~km}$. The Near detector (of size $4 \times 4 \times 15 \mathrm{~m}$ ) is located $100 \mathrm{~m}$ underground, in Fermilab, while the Far detector is 16x16x60m large, located on the surface, covered by $1.2 \mathrm{~m}$ of concrete and $15 \mathrm{~cm}$ of barite. Timing resolution, and the size of the far detector, allow for the separation of the upward-going charged tracks (muons) from a huge downgoing flux $(140 \mathrm{kHz})$ of the cosmic muons. Some of the upward going muons can originate from DM (particle-antiparticle) annihilations, possibly occuring in the Sun. A dedicated trigger software module selects the upward-going tracks from the Far detector data stream. Using the angular position of the Sun, the upwardgoing backround of tracks, originating from the atmospheric neutrinos, can be separated from the anticipated DM annihilation signal: the high-energy neutrinos born in the Sun, transmuting into the charged lepton via weak charged current interaction, just below the detector [11]. WIMP-like dark matter particles, eventualy captured by the Sun, are not expected to be lightweight, and one usually assumes $M_{\chi}>2 \mathrm{GeV} / \mathrm{c}^{2}$. The upward-going muon data from the NOvA Far detector will be accumulated until 2025.

Another DM-hunting effort with the NOvA detectors has been initiated recently, in the remote connection with the search for the faint signals, or the neutrino bursts, possibly correlated with the obseved gravitational wave events [12]. The effort is motivated by the gravitational focussing [13] hypothesis, of the slow DM tidal streams in the galactic (Milky Way) DM halo [14], by the Sun, or by other massive members of our Solar system - e.g. Jupiter. A faint anomalous signals (possibly including the increased electronic noise) may be carefully analyzed as a potential evidence for a DM-related interactions with the NOvA experimental equipment, if they are statistically significant.

\section{ACKNOWLEDGEMENTS}

This work was supported by the Ministry of Education, Youth and Sports of the Czech Republic, under Operational Programme Research, Development and Education project EF16_013/0001787, and the Large Research Infrastructure project LM2015068. Hospitality of the Fermilab personnel and NOvA scientists, and support from the Interexcellence project LTT18001 are also appreciated.

\section{References}

[1] G. Bertone and D. Hooper, Reviews of Modern Physics 90, 045002 (2018).

[2] T.G. Rizzo, Physical Review D99, 115024 (2019).

[3] R. Bernabei et al., European Physics Journal C56, 333 (2008).

[4] P. deNiverville and C. Frugiuelle, Physical Review D99, 051701R (2019).

[5] J. Bian, arXiv:1710.03428, Proceedings of the 2017 APS DPF Meeting, July 31 - August 4, 2017, SLAC eConf C170731.

[6] P. Adamson et al., Nuclear Instruments and Methods in Physics Research A806, 279 (2016).

[7] T. Kitahara, T. Okui, G. Perez, Y. Soreq, K. Tobioka, Physical Review Letters 124, 071801 (2020).

[8] B. Wang et al., Journal of Physics: Conference Series 888, 012123 (2017).

[9] V. de Romeri, K.J. Kelly, and P.A.N.Machado, Physical Review D100, 095010 (2019).

[10] E. Angelico J. Eisch, A. Elagin, H.J. Frisch, S. Nagaitsev, and M. Wetstein, Physcal Review D100, 032008 (2019).

[11] K. Choi et al. (Super-Kamiokande Collaboration), Physical Review Letters 114, 141301 (2015).

[12] M.A. Acero et al. (NOvA Collaboration), arXiv:2001.07240 [hep-ex] (2020).

[13] B.R. Patla, R.J. Nemiroff, D.H.H. Hoffmann, and K.Zioutas, The Astrophysical Journal 780:158 (2014).

[14] A. Bonaca et al., The Astrophysical Journal 795:94 (2014). 